\title{
MS38-05 | Combining a Nine-Crystal Multianalyser Stage with a Hybrid CdTe Photon Counting Detector for High-Resolution X-Ray Powder Diffraction at
}

\section{ESRF-ID22}

Dejoie, Catherine (ESRF, Grenoble, FRA); Autran, Pierre-Olivier (ESRF, Grenoble, FRA); Covacci, Ezio (ESRF, Grenoble, FRA); Fitch, Andrew (ESRF, Grenoble, FRA)

The high-resolution powder diffraction beamline ID22 at ESRF combines a wide choice of photon energies with high brightness, allowing high-flux, high-resolution powder diffraction measurements at wavelengths down to 0.3 Å. In routine operation, a bank of nine scintillation detectors is scanned to record the diffracted intensity versus 2theta, each detector preceded by a Si 111 analyzer crystal. Although the current system has operated successfully for the past twenty years, recent developments in detector technology could be exploited to improve performance. To do so, a Pilatus $3 \mathrm{X}$ CdTe $300 \mathrm{~K}-\mathrm{W}$ was mounted on the arm of the diffractometer, replacing the nine scintillator detectors, and used for standard continuous-scanning acquisition. This arrangement offers major advantages in terms of data handling and processing. By varying the axial width of a region of interest as a function of 2theta, peak widths, peak shapes, and the statistical quality of the data, particularly at high angles, can be improved. In addition, an analyzer crystal maps a position in the sample directly onto a position on the detector, thus conferring spatial/depth resolution to the measurement. Even greater improvements are expected in the future by exploiting the smaller pixel size and the higher speed of the Eiger $2 \mathrm{X}$ CdTe $2 \mathrm{M}$ detector, leading to the possibility of 3-dimensional mapping of complex systems during in-situ and time-resolved experiments. Combining the high efficiency of a hybrid photon-counting area detector with the high angular resolution given by analyzer crystals is an innovative approach to improving the overall performance of high-resolution powder diffraction. 